\title{
Un análisis sobre la percepción normativa, y su relación con los valores y el bienestar psicosocial
} An analysis of normative perception, and its relation to values and psychosocial well-being

\author{
Luciana Saggese ${ }^{1}$, ORCID 0000-0001-6659-2721 \\ Maite Beramendi ${ }^{2}$, ORCID 0000-0002-6113-5529 \\ ${ }^{12}$ Universidad de Buenos Aires. Argentina
}

\begin{abstract}
Resumen: Diversos autores coinciden en que la transgresión en la Argentina es una práctica común, arraigada y naturalizada. Se propone un modelo que analiza la percepción del sistema normativo como un organismo complejo que comprende no solo las normas en sí, sino a las instituciones y agentes que intervienen en él. El objetivo del presente estudio es analizar, desde una perspectiva psicosocial, la relación entre la percepción del sistema normativo, el bienestar y los valores de trabajadores de la Ciudad Autónoma de Buenos Aires (CABA) y el Conurbano Bonaerense. Se diseñó un estudio no-experimental, descriptivo-correlacional. La muestra es intencional, compuesta por 148 trabajadores. Los análisis descriptivos indican una mala percepción del sistema normativo, priman valores asociados a la salud, la supervivencia y la estabilidad personal; y un alto nivel de bienestar psicosocial. Como hallazgo novedoso no se encontraron relaciones entre la percepción del sistema normativo y el resto de las variables.
\end{abstract}

Palabras clave: bienestar, normas, trabajadores, trasgresión, valores

Abstract: Several authors agree that transgression in Argentina is a common practice rooted and naturalized. , It arises a model which analyzes the perception of the legal system as a complex organism comprising not only the norms themselves, but the institutions and actors involved in it. The objective of the present study is to analyze, from a psychosocial perspective, the relationship between the perception of the normative system, the well-being and the values of workers of the City of Buenos Aires and the Conurbano Bonaerense. A non-experimental, descriptive-correlational study was designed. The sample is intentional, composed of 148 workers. Descriptive analyzes indicate a poor perception of the normative system, values associated with health, survival and personal stability prevail, and a high level of psychosocial well-being. As a novel finding, relations between the negative perception of the normative system and the rest of the variable were not found.

Key words: norms, transgression, values, well-being, workers

Recibido: 26/10/2018

Aceptado: 18/06/2019

Cómo citar este artículo:

Saggese, L., \& Beramendi, M. (2019). Un análisis sobre la percepción normativa, y su relación con los valores y el bienestar psicosocial. Ciencias Psicológicas, 13(2), 333-345. doi: 10.22235/cp.v13i2.1890

Correspondencia: Maite Beramendi, Universidad de Buenos Aires. Argentina, Email maiteberamendi@gmail.com 
En los últimos años, se ha podido visualizar un incremento en la trasgresión normativa en la Argentina. La misma aparece hoy en día como una práctica común y arraigada dado que se visualizan cotidianamente todo tipo de transgresiones que van desde el incumplimiento de normas de convivencia hasta actos de corrupción política (Beramendi 2014; Beramendi \& Zubieta, 2013, 2014, 2015, 2016, 2018). Esto ha permitido hablar de una cultura de la trasgresión argentina que da cuenta de prácticas y creencias que perpetúan el incumplimiento normativo (Beramendi, 2014; Nino, 2005). A partir de altos índices de trasgresión y los factores que influyen en la percepción de falta de legitimidad institucional, surge la necesidad de indagar la relación de los individuos con las normas y sus instituciones, no sólo a nivel individual sino desde un abordaje que contemple una perspectiva psicosocial más compleja (Beramendi y Zubieta, 2014, 2018). Dentro de este complejo entramado que relaciona al individuo y a la sociedad, se configuran valores, individuales y colectivos que se materializan en normas, y funcionan como marcos de referencia en la organización y control de las dinámicas sociales e institucionales (Gouveia, Milfont, Fisher y Santos, 2008; Gouveia, Milfont y Guerra, 2014). Estos valores, al guiar la conducta y funcionar como representaciones cognitivas tanto de las necesidades humanas como de las demandas institucionales y societales (Gouveia et al., 2008; Gouveia, Milfont, Fisher y Coelho, 2009), influyen en la percepción del sistema normativo - y el consecuente accionar -, y estos ambos, debido a su impacto personal, influyen en el bienestar (Schwartz, 2012).

\section{Transgresión Normativa}

En el marco de la psicología social, Beramendi (2014) y Beramendi y Zubieta (2014, 2018) proponen un modelo para analizar el funcionamiento del sistema normativo, definido como un organismo complejo que comprende a las normas, a las instituciones y a los agentes que las promueven, respaldan y controlan, así como a las creencias y prácticas normativas de los ciudadanos sobre las normas. El sistema normativo contempla tres dimensiones:

1. La percepción de legitimidad: es una evaluación que se realiza sobre la percepción de justicia en la organización de las instituciones, la gestión de las autoridades y la distribución social de los recursos. La falta de legitimidad conduciría a que las personas tiendan a no aceptar voluntariamente las normas, porque creen que no es "correcto" o “justo". Esto promueve la inestabilidad de cualquier estructura institucional. Habría seis atributos que contribuirían a esta falta de legitimidad: percepción negativa de la distribución económica, percepción negativa del funcionamiento de los procesos de justicia, baja confianza institucional, percepción de corrupción, percepción de ineficacia institucional, y el grado de autoritarismo por parte de las autoridades.

2. La percepción de transgresión: es una evaluación sobre la percepción de creencias y estándares de conductas transgresoras en una sociedad, que no son congruentes con lo socialmente esperado. Para ello se contemplan cinco atributos que posibilitarían una mayor percepción de transgresión social: el grado de falta de sanción y control normativo, asociado a la creencia de que ante la falta de control o sanción por parte de las autoridades competentes las personas tenderán a incumplir las normas, la habituación, la generalización y la naturalización sobre el incumplimiento normativo, y, finalmente, si las personas desarrollan sistemas normativos individuales según sus propios valores, costumbres y/o normas individuales.

3. La percepción de debilidad normativa: es una valoración negativa que se realiza sobre el poder de la norma determinado la obediencia y credibilidad de las normas que se deberían cumplir. Se realiza a partir de tres atributos; la arbitrariedad, la organización y gestión de las instituciones a partir de dobles estándares donde las normas informales tienen más poder que las formales, y el poder de la 
autoridad por sobre el poder de la norma.

Diversas investigaciones fueron realizándose a partir de este enfoque, que analizaron la percepción del sistema normativo y distintas variables. Se realizó un estudio transcultural donde se compara a ciudadanos argentinos, peruanos y venezolanos obteniendo como resultado que la percepción del sistema normativo es más negativa en Venezuela, seguida por Perú y finalmente la Argentina (Beramendi, Espinosa y Acosta, 2019). En el contexto local, una investigación arroja que en Argentina emerge principalmente la falta de legitimidad institucional, la cual se relaciona directamente con la transgresión - hecho naturalizado y aceptado - y la percepción de un doble estándar de normas formales e informales que coexisten. Y se encontró que las mujeres tienen una percepción negativa mayor que los hombres del sistema normativo (Beramendi, 2014). Asimismo, en Buenos Aires, estudiantes universitarios y futuros oficiales del ejército Argentino percibieron que el funcionamiento del sistema normativo es adverso, y se asocia con sentimientos de desmoralización social, y emociones/sentimientos negativos como tristeza y desconfianza (Beramendi y Zubieta, 2016).

\section{Valores}

Una de las teorías más reconocidas de valores en la actualidad es la teoría Teoría Fumcionalista de los Valores Humanos (Gouveia et al. 2008). Esta presentaun modelo integrador que asume cuatro supuestos principales: 1) Naturaleza Humana: toma la naturaleza benevolente o positiva de los seres humanos, de manera que sólo admite valores positivos. 2) Base Motivacional: se asumen a los valores como representaciones cognitivas de las necesidades humanas pero también de demandas institucionales y societales. 3) Carácter Terminal: únicamente los valores terminales han sido tenidos en cuenta, concebidos como metas cognitivas superiores y transcendentes, no restringiéndose a metas inmediatas y biológicamente urgentes. 4) Principios-Guía Individuales: valores incorporados por la cultura que han sido útiles a la supervivencia del grupo, haciéndolos deseables, y que posibilitan la continuidad de la sociedad, posibilitando categorías generales de orientación para las conductas de los individuos.

Gouveia, Santos, Athsyde, Souza y Gusmão (2014) han identificado dos funciones principales de los valores humanos: (1) guían las acciones (tipo de orientación) y (2) representan las necesidades humanas (tipo de motivador). La primera función presenta tres posibilidades de orientación: social, central y personal. La segunda función de los valores es representar cognitivamente las necesidades humanas. En este sentido, todos los valores pueden ser clasificados como materialistas (pragmáticos) o humanitarios (idealistas). Los valores materialistas están relacionados con ideas prácticas, y una orientación hacia metas específicas y reglas normativas. Los valores humanitarios expresan una orientación universal, basada en ideas y principios más abstractos. De esta manera el eje horizontal correspondería a la función de los valores para guiar acciones humanas, mientras que el eje vertical indicaría la función de los valores para representar cognitivamente las necesidades humanas.

De acuerdo con la Figura 1, los tres tipos de orientación son representados por dos subfunciones cada una: social (normativa $e$ interactiva), central (existencia $y$ suprapersonal) y personal (realización y experimentación). De modo similar, tres subfunciones representan cada uno de los tipos motivadores: materialista (existencia, normativa y realización) y humanitario (suprapersonal, interactiva y experimentación). Por lo tanto, es posible hablar de seis cuadrantes diferenciados: socialmaterialista, central-materialista, personalmaterialista, social-humanitario, centralhumanitario y personal-humanitario (Gouveia, Milfont et al., 2014). 

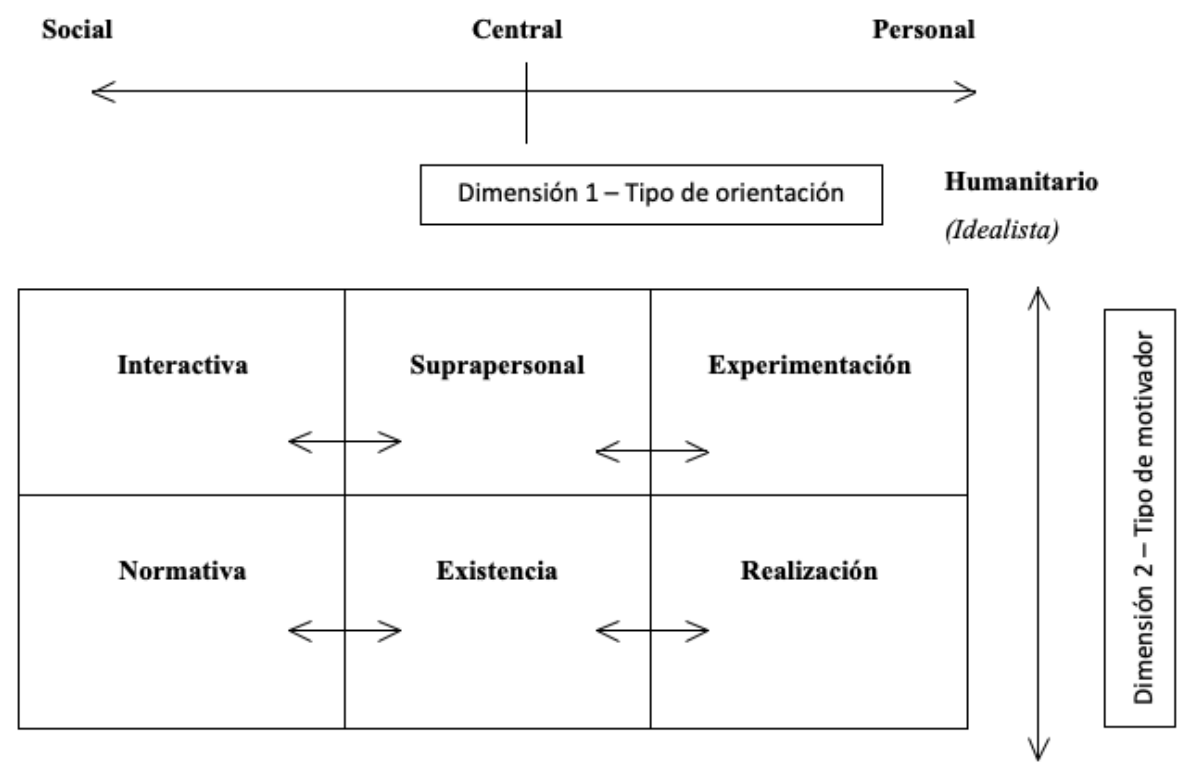

(Pragmático)

Materialista

Figura 1.

Facetas, dimensiones y subfunciones de los valores básicos (Gouveia, 2013)

Tanto las funciones como subfunciones de los valores son estructuras latentes, que precisan una representación a través de indicadores o valores específicos. Gouveia, Milfont et al. (2014) describen seis subfunciones:

a. Subfunción Existencia. Representa el motivador materialista y representa una orientación central. Salud, supervivencia y estabilidad personal son valores que pueden representar esta subfunción.

b. Subfunción Realización. Valores con origen en un principio personal para guiar la vida de los individuos, mientras ponen énfasis en realizaciones materiales (e.g., éxito, prestigio y poder).

c. Subfunción Normativa. Motivador materialista con una orientación social, reflejando la necesidad de control, preservar la cultura y las normas convencionales (e.g., tradición, obediencia y religiosidad).

\section{d. Subfunción Suprapersonal.} Representan las necesidades estéticas, de cognición, y autorrealización (e.g., conocimiento, madurez y belleza). Definidos como humanitarios, orientados por un tipo central.

e. Subfunción Experimentación. La necesidad fisiológica de satisfacción o la inclinación hacia el principio de placer (hedonismo) (e.g., sexualidad, placer y emoción). Motivador humanitario, pero con una orientación personal.

f. Subfunción Interactiva. Motivador humanitario, pero con una orientación social. El destino común, la experiencia afectiva entre individuos, y las necesidades de pertenencia (e.g., afectividad, convivencia y apoyo social).

\section{Bienestar}

La perspectiva psicológica y social de la salud y el bienestar conciben un abordaje que da cuenta de la interrelación de necesidades individuales y sociales como promotores del bienestar o el malestar. Se trata de estudiar no sólo los criterios psicológicos sino también los criterios sociales del bienestar, esto es, la relación de las personas 
con su medio y de cómo éste les asegura su bienestar, de criterios relacionales y microsociales que la sociedad debe ofrecer a la persona para que ésta logre satisfacer sus necesidades (Hervás y Vázquez, 2013).

La necesidad de medidas breves y exhaustivas que puedan utilizarse para realizar evaluaciones rápidas, fiables y válidas sobre el bienestar promovieron el desarrollo del Modelo Integrativo de Bienestar, The Pemberton Happiness Index (PHI) (Hervás y Vázquez 2013) validado para una variedad de idiomas y países desde su inicio. Hervás y Vázquez (2013) propusieron un abordaje integral el cual incorpora tres tipos de bienestar: subjetivo, psicológico y social.

El bienestar subjetivo: se define como "una amplia categoría de fenómenos que incluye las respuestas emocionales de las personas, la satisfacción con los dominios y los juicios globales sobre la satisfacción con la vida" (Diener, Suh, Lucas y Smith, 1999, p.277). Se vincula con la tradición hedónica de bienestar, la cual atribuye que el bienestar consiste en la felicidad subjetiva, y se refiere a la experiencia de placer versus displacer.

El bienestar psicológico: Ryff y Keyes (1995) centraban su atención en el desarrollo de las capacidades y el crecimiento personal, concebidas como principales indicadores del funcionamiento positivo, tanto de la percepción personal e interpersonal, la apreciación del pasado, la participación en el presente y la movilización para el futuro (Valle, Beramendi y Delfino, 2011). Vinculado con la tradición eudaemónica del bienestar, el cual señala que la verdadera felicidad se encuentra en la expresión de la virtud; en hacer lo que vale la pena hacer (Ryan y Deci, 2001), como también en vivir una vida que represente la excelencia humana (Ryan, Huta y Deci, 2006).

El bienestar social tiene como propósito caracterizar el funcionamiento positivo, no a nivel privado y personal, sino al nivel de la relación del individuo con el dominio público y social (Keyes, 1998). Es decir, darle relevancia tanto a "lo individual como a lo social, al mundo dado como al mundo intersubjetivamente construido, a la naturaleza y a la historia" (Blanco y Diaz, 2005, pág. 585). Keyes (1998) señala que la evaluación que cada uno hace de las circunstancias (bienestar social), y el funcionamiento de la sociedad también es necesario para pensar y comprender el concepto de bienestar.

\section{Objetivos}

El objetivo general de la presente investigación es analizar la relación entre la percepción del sistema normativo, el bienestar psicosocial, y los valores humanos en trabajadores del ámbito privado y público de la Ciudad Autónoma de Buenos Aires y el Conurbano Bonaerense. Como objetivos específicos se espera describir la percepción del sistema normativo, el bienestar psicosocial, $\mathrm{y}$ los valores humanos en trabajadores del ámbito privado y público de la Ciudad Autónoma de Buenos Aires y el Conurbano Bonaerense. Y por otro lado, indagar perfiles diferenciales en los aspectos psicosociales mencionados en el objetivo uno en función de aspectos sociodemográficos, tales como la edad, el género, el lugar de residencia, la práctica religiosa, el ámbito de trabajo y aspectos psicosociales como la autopercepción económica y el auto-posicionamiento ideológico.

\section{Método}

\section{Diseño y Tipo de estudio}

El diseño del estudio es no experimental, transversal, siendo un tipo de estudio descriptivo-correlacional.

\section{Participantes}

La muestra fue no-probabilística e intencional, y compuesta por 148 trabajadores residentes en la Ciudad Autónoma de Buenos Aires (40.5\%) y el Conurbano Bonaerense (59.5\%). El 33.85\% ( $n=50)$ pertenece al género masculino, y el 66.2\% (n=98) al género femenino, con un promedio de edad de 31.40 años $(D E=10.99 ; \quad M i n=20$, Máx=77). Las 
organizaciones en las cuales trabajan los participantes se ubican un $66.9 \%$ en CABA y el $33.1 \%$ en el Conurbano Bonaerense. Las mismas se diferencian entre instituciones de ámbito público, el 18.9\% (n= 28), y de ámbito privado, el $81.1 \%(n=120)$. El 35.8\% expresa trabajar entre un rango de 10 y 5 años, seguido por el $31.1 \%$ que expresa trabajar hace menos de 5 años. El promedio general de tiempo de trabajo en la organización actual es de 5.83 años $(D E=8.30)$.

\section{Instrumentos}

Se diseñó un cuestionario autoadministrado compuesto por las siguientes escalas, y un conjunto de preguntas psicosociales:

Escala de Percepción del Sistema Normativo (EPSN, Beramendi y Zubieta, 2014): compuesta por 20 ítems que se organizan en tres dimensiones que evalúan la Percepción de Falta de Legitimidad, la Percepción de Transgresión y la Percepción de Debilidad Normativa. Se presentan ítems tales como "los organismos públicos son poco eficientes" o "muchas normas son arbitrarias y sin sentido", cuyas respuestas se presentan en un formato de escala tipo Likert, de 1 (totalmente en desacuerdo) a 7 (totalmente de acuerdo). Las puntuaciones elevadas dan cuenta de una percepción negativa del sistema normativo. La consistencia interna de la escala que fue aplicada en este estudio fue aceptable $(\alpha=.86)$,

Cuestionario de los Valores Básicos (CVB, Gouveia et al., 2014): la versión más actual está formada por 18 ítems/valores. El cuestionario presenta dos descripciones para cada valor (por ejemplo, EMOCIÓN: disfrutar con desafíos o situaciones desconocidas; buscar aventuras /TRADICIÓN: seguir las normas sociales de su país; respetar las tradiciones de su sociedad). Se evalúa cada ítem en una escala de siete puntos, con los siguientes extremos: 1 (Totalmente sin importancia) y 7 (De máxima importancia), considerando su importancia como principio que guía sus vidas. La consistencia interna de las dimensiones de la escala que fueron aplicadas en esta toma fueron aceptables: experimentación ( $\alpha=.53)$, realización $(\alpha=.42)$, existencia $(\alpha=.31)$, suprapersonal $(\alpha=.45)$, interactiva $(\alpha=.31)$ y normativa $(\alpha=.54)$. Pemberton Happiness Index (PHI, Hervás y Vázquez, 2013): diseñada para medir la felicidad en la población general, consta de 11 ítems relacionados con el bienestar recordado, cada uno con una escala de Likert de 11 puntos; donde 0 es totalmente en desacuerdo y 10 totalmente de acuerdo. La escala de bienestar recordada de la PHI se considera unidimensional. La puntuación de bienestar recordada se calcula con la puntuación media de los 11 ítems y puede variar de 0 a 10 . La consistencia interna de la escala que fue aplicada en esta toma fue aceptable $(\alpha=.87)$.

Preguntas sociodemográficas: edad, género, ciudad de residencia, localización de la organización en la que trabaja.

\section{Procedimiento}

La recolección de datos se realizó a partir de dos modalidad: versión on-line y en versión papel. La versión por internet se realizó a partir de la plataforma SurveyMonkey. Para la versión papel, la investigadora principal se acercó a empresas privadas y públicas para poder aplicar el cuestionario. Las administraciones fueron individuales.

La recolección de datos se llevó a cabo desde noviembre de 2016 a febrero de 2017. A todos los participantes se les brindó un consentimiento informado que explicitaba la participación era voluntaria, que la información era confidencial y que sólo se usaría para fines académicos. Los análisis de los datos se realizaron a partir del programa estadístico SPSS 21.

\section{Resultados}

\section{Análisis descriptivo de las escalas}

A partir de los datos obtenidos, como se observa en la Tabla 1, los participantes perciben negativamente el sistema normativo 
argentino. La dimensión peor evaluada fue la Percepción de la Falta de Legitimidad, seguida por la Percepción de Transgresión, y por último por la dimensión de Percepción de Debilidad Normativa. Para comparar las puntuaciones entre las dimensiones, se calculó un ANOVA de medidas repetidas. Los datos indican que se rechaza la prueba de esfericidad de Mauchly, por lo cual se utiliza la corrección de Greenhouse-Geisser, la cual indica que hay una diferencia entre las dimensiones, $F(1.915)=17.303, p<.001$. Para observar las diferencias entre estas, se comparó los efectos principales, corroborando las diferencias entre todas las variables de la escala y el orden nombrado.

Tabla 1.

Análisis descriptivos de las escalas

\begin{tabular}{lll}
\hline & $M$ & $D E$ \\
\hline Escala de Percepción del Sistema Normativo & & \\
\hline Dimensión Falta de Legitimidad & 5.55 & .89 \\
Dimensión Percepción de Transgresión & 5.29 & 1.14 \\
Dimensión Debilidad Normativa & 5.03 & 1.10 \\
EPSN & 5.29 & .85 \\
\hline Escala de Valores Básicos & & \\
\hline Subfunción Experimentación & 4.96 & .83 \\
Subfunción Realización & 4.25 & .88 \\
Subfunción Existencia & 5.52 & .74 \\
Subfunción Suprapersonal & 5.18 & .81 \\
Subfunción Interactiva & 5.27 & .79 \\
Subfunción Normativa & 4.01 & 1.18 \\
\hline PHI & 8.01 & 1.33 \\
\hline
\end{tabular}

Nota: el rango de respuesta de la Escala de Percepción del Sistema Normativo es $=7$.

Del Cuestionario básico de valores es $=7, \mathrm{y}$ del Bienestar integrativo es un rango de 0 a 10

En relación a los resultados de la escala de Valores Básicos, la Tabla 1 exhibe los análisis descriptivos que indican las puntuaciones obtenidas por los participantes. Al igual que en el anterior análisis, se llevó a cabo un ANOVA de medidas repetidas, que rechazó la prueba de esfericidad de Mauchly, indicando que hay diferencias entre las subfunciones, $F(4,173)=79.806, p<.001$. Asimismo, para observar las diferencias entre las subfunciones, se comparó los efectos principales. Esto dio como resultado que la subfunción existencia obtuvo las puntuaciones más altas. A continuación, la subfunción interactiva y la Suprapersonal también reflejaron puntuaciones elevadas. Mientras que las subfunciones experimentación, realización y normativa arrojaron valoraciones más bajas, siendo esta última la que obtuvo menor puntuación.

Con respecto a la Escala de Bienestar Integrativo (PHI), la puntuación de la muestra indica que los participantes tienen un bienestar elevado. En este sentido, tanto el bienestar subjetivo, como el psicológico y el social se encuentran integrados permitiendo un funcionamiento óptimo de la persona como de interacción con el contexto social (Tabla 1). 
Comparación entre variables asociadas al trabajo

En relación a las variables asociadas al trabajo, podemos observar en la Tabla 2 que no se observa una diferencia en la percepción del sistema normativo según el ámbito de trabajo de los participantes. En lo que respecta a la comparación de los valores se puede observar que la única diferencia se encuentra en la subfunción Suprapersonal donde los participantes del ámbito privado poseen puntuaciones más altas. No se hallaron diferencias significativas respecto a la residencia del lugar de trabajo.

Tabla 2 .

Ámbito de trabajo

\begin{tabular}{lcccc}
\hline & $\begin{array}{c}\text { Público } \\
M d(D E)\end{array}$ & $\begin{array}{c}\text { Privado } \\
M d(D E)\end{array}$ & & $p$ \\
\hline EPSN & $5.23(1.09)$ & $5.38(.76)$ & 1288.5 & .06 \\
\hline Subfunción Experimentación & $5.00(1.04)$ & $4.95(.78)$ & 1587 & .64 \\
Subfunción Realización & $4.00(.75)$ & $4.28(.91)$ & 1511.5 & .41 \\
Subfunción Existencia & $5.66(.76)$ & $5.53(74)$ & 1609 & .73 \\
Subfunción Suprapersonal & $5.00(.81)$ & $5.25(.80)$ & 1239.5 & .03 \\
Subfunción Interactiva & $5.16(.73)$ & $5.31(.80)$ & 1368.5 & .12 \\
Subfunción Normativa & $4.00(1.11)$ & $4.12(1.20)$ & 1570 & .59 \\
\hline PHI & $8.09(1.02)$ & $8.02(1.39)$ & 1521 & .44 \\
\hline
\end{tabular}

Por otro lado, en relación a los grupos diferenciados que representan distintos rangos de años de trabajo se hallaron diferencias, donde las personas que trabajan desde hace 5 a 10 años tienen puntuaciones más altas en la subfunción experimentación $\quad(\mathrm{z}=10.840$, $p=.03)$.

Ahora bien, en cuanto al análisis de las variables sociodemográficas evaluadas, la
Tabla 3 muestra que se hallaron diferencias respecto al género, particularmente en las subfunciones suprapersonal e interactiva, siendo éstas mayormente valoradas por las mujeres. Asimismo se hallaron diferencias relevantes en cuanto al bienestar de los trabajadores, donde se observa que las mujeres perciben un menor bienestar en relación a los hombres.

Tabla 3.

Diferencia de género

\begin{tabular}{lccccc}
\hline & $\begin{array}{c}\text { Femenino } \\
M(D E)\end{array}$ & $\begin{array}{c}\text { Masculino } \\
M(D E)\end{array}$ & $t$ & $g l$ & $P$ \\
& $5.30(.82)$ & $5.29(.82)$ & -.071 & 88.73 & .94 \\
\hline EPSN & $4.90(.73)$ & $5.09(1.01)$ & 1.13 & 76.37 & .26 \\
Subfunción Experimentación & $4.16(.84)$ & $4.44(.73)$ & 1.77 & 88.00 & .67 \\
Subfunción Realización & $5.50(.72)$ & $5.57(.80)$ & .42 & 89.90 & .67 \\
Subfunción Existencia & $5.29(.76)$ & $4.98(.90)$ & -2.07 & 85.23 & .03 \\
Subfunción Suprapersonal & $5.37(.71)$ & $5.10(.91)$ & -1.86 & 79.94 & .05 \\
Subfunción Interactiva & $4.17(1.11)$ & $3.98(1.33)$ & -.88 & 84.46 & .35 \\
Subfunción Normativa & $8.17(1.34)$ & $8.70(1.27)$ & -2.07 & 103.86 & .04 \\
\hline PHI & & & & & \\
\hline
\end{tabular}


En relación a las diferencias según la edad de los participantes solo se observaron diferencias en los valores. A partir la prueba de Kruskal-Wallis y el análisis de contraste Bonferroni, se halló que los participantes más jóvenes presentan mayormente valores de experimentación que los grupos mayores $(z=$ 11.29, $p=.01$ ). Además, se encontró diferencias entre los grupos de mayor edad y mediana edad siendo el primer grupo quien presenta puntuaciones más altas $(z=7.18, p=$ .03). En relación a la dimensión normativa se observa que sólo hay diferencias entre el grupo de mediana edad y el más pequeño, siendo los primero quienes tienen mayores puntuaciones $(\mathrm{z}=7.87, p=.02)$.

Por otro lado, se hallaron diferencias entre los trabajadores que residen en CABA y el Conurbano Bonaerense, siendo estos últimos quienes tienen menores puntuaciones en la dimensión normativa, $t(146)=2.264, p=$ $.03 ; M_{C A B A}=4.37, D E=1.24 ; M_{\text {Conurbano }}=3.93$, $D E=1.12)$. En lo que respecta al nivel educativo, se reagrupó esta variable sociodemográfica, y se obtuvo una diferenciación de la variable entre primario, secundario, terciario y universitario. Los resultados arrojan que no se hallaron diferencias entre las variables analizadas y el nivel educativo de los trabajadores.

\section{Relación entre las variables}

Para analizar si había una relación entre la percepción del sistema normativo, los valores y el bienestar, se realizó un análisis de correlación de Pearson. No se halló una relación entre la percepción del sistema normativo, los valores y el bienestar. En cambio, se halló una relación entre los valores y el bienestar. Más precisamente entre la subfunción suprapersonal y el PHI $[r(148)=$ $.18, p=.03]$, y la subfunción interactiva y el PHI, $r(148)=.18, p=.03$.

\section{Discusión}

Los resultados revelan que no hay una relación entre la percepción del sistema normativo, los valores y el bienestar. Estos datos llaman la atención ya que estos conceptos son entendidos como parte de un sistema que se interrelaciona e influye mutuamente, y como parte del entramado social que converge en la creación de identidad cultural e individual. Entendiendo por este hecho que, ya sea positiva o negativamente, se hallaría relación entre las variables como parte de la complejidad que hace a la sociedad.

Estudios previos (Gouveia et al., 2008, Gouveia, Milford et al., 2014; Schwartz, 1977) sostienen que los valores al estar comprometidos de manera holística con las personas inciden en su manera de percibir la norma, y esto mismo afectará el bienestar. Pero, es probable que un contexto donde la transgresión forme parte del proceso de socialización, histórica y culturalmente arraigada, influyendo directamente en la legitimidad de las instituciones, ya no repercuta en otros procesos complejos del individuo debido a que se encuentra naturalizada.

Por otro lado, se desprende de los resultados una relación entre el bienestar y los valores de los trabajadores. La integración entre valores profundamente arraigados, que están comprometidos de manera holística o total en la vida del individuo, permite el desarrollo óptimo de las capacidades psicologías, y de la salud, tanto como del pensamiento cognitivo (Ryff y Keyes, 1995). Los resultados arrojaron más precisamente una relación entre el bienestar y valores como conocimiento, madurez, belleza, afectividad, convivencia y apoyo social. Estos valores son principalmente representados por jóvenes (Gouveia, Santos et al., 2014), y se caracterizan por ser valores que responden a una motivación más idealista o humanitaria donde no repercute si hay acceso o no a los bienes materiales necesarios y básicos para la supervivencia, que sí se asocian más con la incidencia de las normas sociales para acceder a ellos. Esta investigación estuvo principalmente formada por jóvenes, lo cual explicaría los resultados.

Los hallazgos muestran que los trabajadores creen en mayor medida que hay 
una falta de legitimidad asociada a la falta de justicia distributiva y procedimental, eventos de corrupción, baja eficacia y confianza institucional, y autoritarismo por parte de las autoridades. En segunda instancia, los participantes perciben altos niveles de transgresión generalizados que se enraízan en creencias y pautas compartidas, así como también visualizan bajos niveles de control y sanción con respecto al incumplimiento de las normas. La transgresión aparece como una creencia compartida y naturalizada en el contexto argentino, lo cual alerta e induce a repensar el concepto de norma, ya que posee un cariz negativo, asociado a la arbitrariedad y al sin sentido. Finalmente, en menor medida, los trabajadores creen que las normas son arbitrarias y sin sentido, y perciben dobles estándares institucionales, donde las normas informales tiene más poder que las formales sugiriendo que la transgresión se introduce en la vida institucional de la sociedad argentina imponiendo pautas de interacción que conviven con ella. Como también creen que las autoridades de las instituciones están por encima de las normas. De esta manera, la gente visualiza la transgresión no como un hecho aislado y reducido a un grupo específico de la sociedad, sino como algo que se plasma de manera generalizada y enraizada en el entramado social. Estos resultados son consistentes con investigaciones previas realizadas en el contexto local (Beramendi, 2014; Beramendi et al., 2019; Beramendi y Zubieta, 2014, 2018).

Respecto a las repercusiones psicosociales los trabajadores manifiestan un tipo de orientación central de los valores, y el tipo de motivador tiene una tendencia a ser mayormente humanitario (Gouveia et al., 2014), lo cual no quiere decir que los participantes sean mejor representados por valores materialistas, o no se pueda hablar de una tendencia hacia lo social o personal. Dicho esto, se pudo observar que la salud, la supervivencia y la estabilidad personal son los valores más estimados, representando una orientación central con un motivador materialista. Los cuales son preferidos por individuos en contextos de escasez económica, o por quienes han sido socializados en tales ambientes (Inglehart, 1977). Ahora bien, los participantes también destacaron las relaciones iguales entre pares, el principio universal de orientación y la apertura al cambio que representan una motivación humanitaria. Usualmente son los jóvenes, mayoría en esta investigación, quienes se caracterizan por este tipo de motivador, donde la igualdad de grupos $\mathrm{y}$ el cambio son algunos valores que los representan. $\mathrm{Y}$ justificando que, contrariamente, la importancia de preservar la cultura y las normas convencionales, valorar la religiosidad y la tradición, sean algunos de los aspectos menos valorados por los participantes. Encontramos que personas mayores son típicamente más proclives a guiarse por estos valores (Rokeach, 1973).

Priorizar valores normativos evidenciaría que la obediencia a la autoridad es importante.

Por otro lado, en lo que respecta al bienestar psicosocial los resultados arrojaron un elevado bienestar en los trabajadores. Esto sugiere un elevado desarrollo de las capacidades y crecimiento personal, concebidas ambas como principales indicadores del funcionamiento positivo de la salud mental (Ryff y Keyes, 1995). Una relación positiva de los trabajadores con su propia satisfacción, las emociones, y un sentimiento de felicidad sustentado en un alto bienestar subjetivo (Rodríguez-Carvajal, Díaz, Moreno-Jiménez, Blanco y van Dierendock, 2010). Resulta llamativo que no una haya incidencia directa del sistema trasgresor sobre el bienestar. Es decir, se contradicen los resultados obtenidos, ya que refieren un elevado bienestar social que implica la relación del individuo con el dominio público y social (Valle et al., 2011), y se obtuvieron elevadas puntuaciones en la valoración negativa que los trabajadores hacen de las circunstancias y el funcionamiento normativo dentro de la sociedad.

Por otro lado, debido a que no se hallaron diferencias en la percepción del sistema normativo entre aquellos que trabajan en el ámbito público del privado, es posible indicar una generalidad en la problemática normativa institucional que atraviesa a toda la 
sociedad y no se circunscribe a un área de la sociedad en particular. A pesar de que no hay diferencia, los trabajadores del sector privado son quienes manifiestan tener un mayor bienestar. En este sentido, se podría pensar que el ámbito privado se caracteriza por un clima organizacional y condiciones laborales más positivas, donde se promueve el desarrollo laboral y personal, la estabilidad, y satisfacción de objetivos en la vida, permitiendo hablar de un funcionamiento psicológico más positivo. $\mathrm{Al}$ ser la transgresión una norma social aceptada y colectivamente aplicada, el incumplimiento normativo se convierte en una norma social descriptiva (Cialdini, 2007), que informa a las personas acerca de cómo deben comportarse normativamente, funciona únicamente como una conducta adaptativa y eficaz al contexto (Beramendi, 2014). Por lo tanto, los trabajadores adoptan, por un lado una manera de manejarse respecto a las normas, disociando su actuar, legitimado por el resto de la sociedad, de su integración personal con la sociedad. En este sentido, se mantiene la autonomía y coherencia socia. El bienestar además de promoverse por el ámbito de trabajo, podría pensarse como resultado de una diferenciación entre el actuar en sociedad y la búsqueda propia e individual de bienestar integral.

En relación al género, se encontró que las mujeres atribuyen mayor importancia que los hombres a valores de conocimiento, madurez y belleza, lo cual se asocia a un contexto cultural actual que le da valor a lo estético y la superación personal. Y al mismo tiempo, representan las necesidades de pertenencia, amor y afiliación, valores que son mayormente socializados, esperados y permitidos en el género femenino. Además, son quienes puntúan más alto en relación al bienestar. Los datos son coherentes con investigaciones previas donde las mujeres miden mejor en relación a los hombres, mostrando tener más satisfecha las necesidades de estabilidad y de confianza (Ryff y Keyes, 1995). Desde una perspectiva social, las mujeres evalúan mejor la calidad de las relaciones que mantienen con la sociedad o sus grupos de pertenencia, a la vez se sienten más útiles, ya que logran sacar más provecho del entorno y aportan al bien común (Zubieta, Muratori y Fernández, 2010).

En la variable edad se constató que los más jóvenes priorizan la satisfacción de las necesidades fisiológicas básicas (e.g., comer, beber, dormir) y la necesidad de seguridad, asociado directamente con el contexto socioeconómico. Además en la sociedad que construyen, los valores ya no están ligados principalmente a la producción. El trabajo ya no lleva consigo los signos del reconocimiento social (Sanchis, 1988) dando lugar a otro tipo de reconocimiento social actual como la búsqueda de felicidad y satisfacción. Asimismo, se observó que este grupo se diferencia de los de mediana edad, quienes se muestran más de acuerdo con la importancia de preservar la cultura y las normas convencionales. Se corresponde con las investigaciones previas, donde personas mayores son típicamente más proclives a guiarse por estos valores (Gouveia et al., 2015). Consecuentemente, son los jóvenes quienes recién comienzan su inserción laboral y quienes trabajan hace 5 a 10 años. Se observa en ellos la necesidad de satisfacción y placer, y la promoción de cambios e innovaciones en la estructura de organizaciones sociales, todas características comúnmente adoptadas por jóvenes.

Una de las posibles limitaciones de la muestra es su carácter no-probabilístico y que sólo se centró en una pequeña parte de la Argentina (CABA y Conurbano). Otra limitación que se halló es la ausencia de contrabalanceo de las escalas.

Estos hallazgos son útiles como herramienta de diagnóstico para comprender las creencias y prácticas que sostienen la transgresión normativa en las instituciones y, en función de ellas, trabajar en intervenciones orientadas a promover su cambio, como en comprender los valores que sostienen dichas prácticas y sus repercusiones en el bienestar de las personas. En próximos estudios se contemplarán estas limitaciones y a la vez se incorporarán nuevas variables de análisis, así como nuevas medidas para evaluar el 
bienestar. Es relevante continuar abordando el fenómeno de la transgresión en el contexto local, pues los niveles de percepción de un mal funcionamiento normativo son altos $y$ perjudican íntegramente al conjunto de la sociedad.

Contribución de los autores:

a) Concepción y diseño del trabajo; b) Adquisición de datos; c) Análisis e interpretación de datos; d)

Redacción del manuscrito; e) revisión crítica del manuscrito.

L.S. ha contribuido en a,b,c,d; M.B. en a, c, d, e.

\section{Referencias}

Beramendi, M. (2014). Percepción del sistema normativo, transgresión y sus correlatos psicosociales en Argentina (Tesis doctoral inédita). Facultad de Psicología. Universidad de Buenos Aires, Ciudad Autónoma de Buenos Aires.

Beramendi, M., Acosta, Y., \& Zubieta, E. (2016). Polarización sociopolítica y percepción del sistema normativo en Venezuela. Psychologia: Avances de la Disciplina, 10(1), 35-45. doi: 10.21500/19002386.2464.

Beramendi, M., Espinosa, A. \& Acosta, Y.(2019). Percepción del Sistema Normativo y sus correlatos psicosociales en Argentina, Perú y Venezuela. Manuscrito enviado para su publicación.

Beramendi, M. \& Zubieta, E. (2013). La identidad nacional y las relaciones sociales en una cultura de la transgresión. Revista de Psicología Política, 13(26), 165-177.

Beramendi, M. \& Zubieta, E. M. (2014). Norma Perversa: transgresión como modelado de legitimidad. Universitas Psychologica, 12(2). doi:10.11144/javeriana.upsy12-2.nptm

Beramendi, M. \& Zubieta, E. M. (2015). Un estudio exploratorio sobre la relación entre la legitimidad institucional y la transgresión normativa en Argentina. Ciencias Psicológicas, 9, 15 - 26.
Montevideo.

Beramendi, M. \& Zubieta, E. (2016). Análisis de la percepción del sistema normativo y sus repercusiones psicosociales en los futuros oficiales del Ejército Argentino. Revista Electrónica de Psicología Política, 12(37), 125-139.

Beramendi, M. \& Zubieta, E. (2018). The factorial validation of the normative system perception scale: a proposal to analyze social transgression. Acta Colombiana en Psicología. 21(1), 249259. doi: 10.14718/acp.2018.21.1.11.

Blanco, A. \& Díaz, D. (2005). El bienestar social: su concepto y medición. Psicothema, 17(4), 582-589.

Cialdini, R. B. (2007). Descriptive Social Norms as Underappreciated Sources of Social Control. Psychometrika, 72(2), 263-268. doi:10.1007/s11336-0061560-6.

Diener, E., Suh, E. M., Lucas, R. E. \& Smith, H. L. (1999). Subjective well-being. Three decades of progress. Psychological Bulletin. 125(2), 276 302.

Gouveia, V. V., Milfont, T. L., Fischer, R., \& Coelho, J. A. P. (2009). Teoria funcionalista dos valores humanos: aplicações para organizações. RAM. Revista de Administração Mackenzie, 10(3), 34-59. doi:10.1590/s167869712009000300004.

Gouveia, V. V., Milfont, T. L., Fischer, R., \& Santos, W. S. (2008). Teoria funcionalista dos valores humanos. In M. L. M. Teixeira (Ed.), Valores humanos e gestão: Novas perspectivas (pp. 47-80). São Paulo, SP: Serviço Nacional de Aprendizagem Comercial. Gouveia, V. V., Milfont, T. L., \& Guerra, V. M. (2014). Functional theory of human values: Testing its content and structure hypotheses. Personality and Individual Differences, 60, 41-47. doi:10.1016/j.paid.2013.12.012.

Gouveia, V. V., Santos, W. S. dos, Athayde, R. A. A., Souza, R. V. L. \& Gusmão, E. É. da S. (2014). Valores, Altruísmo e Comportamentos de Ajuda: 
Comparando Doadores e Não Doadores de Sangue. Psico, 45(2), 209-218. doi:10.15448/1980-8623.2014.2.13837.

Gouveia, V., Milfont, T. L., Correa Vione, K. \& Santos, W. S. (2015). Guiding Actions and Expressing Needs: On the Psychological Functions of Values. Psykhe (Santiago), 24(2), 1-14. doi:10.7764/psykhe.24.2.884.

Hervás, G., \& Vázquez, C. (2013). Construction and validation of a measure of integrative well-being in seven languages: The Pemberton Happiness Index. Health and Quality of Life Outcomes, 11(1), 66. doi:10.1186/1477-7525-11-66.

Inglehart, R. (1977). The silent revolution: changing values and political styles among western publics. Nueva Jersey: Princenton University Press,

Keyes, C. L. M. (1998). Social Well-Being. Social Psychology Quarterly, 61(2), 121-140. doi:10.2307/2787065.

Monsegur, S., Espinosa, A., \& Beramendi, M. (2014). Identidad nacional y su relación con la dominancia social y la tolerancia a la transgresión en residentes de Buenos Aires (Argentina). Interdisciplinaria: Revista de Psicología y Ciencias Afines, 31(1), 523. doi:10.16888/interd.2014.31.1.1.

Nino, C. (2005). Un país al margen de la ley. Buenos Aires: Ariel.

Rokeach, M.(1973). The nature of human values. NewYork: Free Press.

Rodríguez-Carvajal, R.; Díaz, D.; MorenoJiménez, B.; Blanco, A. \& van Dierendock, D. (2010). Vitalidad y recursos internos como componentes del constructo de bienestar psicológico. Psicothema, 22(1), 63-70.Ryan, R. M., Huta, V., \& Deci, E. L. (2006). Living well: a self-determination theory perspective on eudaimonia. Journal of Happiness Studies, 9(1), 139-170. doi:10.1007/s10902-006-9023-4.
Ryan, R. M., \& Deci, E. L. (2001). On Happiness and Human Potentials: A Review of Research on Hedonic and Eudaimonic Well-Being. Annual Review of Psychology, 52(1), 141-166. doi:10.1146/annurev.psych.52.1.141.

Ryff, C. D., \& Keyes, C. L. M. (1995). The structure of psychological well-being revisited. Journal of Personality and Social Psychology, 69(4), 719-727. doi:10.1037/0022-3514.69.4.719.

Sanchis, E. (1988). Valores y actitudes de los jóvenes ante el trabajo. Reis, 41, 131151. doi:10.2307/40183314.

Schwartz, S. H. (1977). Normative influence on altruism. En L. Berkowitz (Ed.), Advances in experimental social psychology (pp. 221-279). New York: Academic Press.

Schwartz, S. H. (2012). An Overview of the Schwartz Theory of Basic Values. Online Readings in Psychology and Culture, 2(1), 1-20. doi: 10.9707/23070919.1116.

Valle, M., Beramendi, M. \& Delfino, G. (2011). Bienestar Psicológico y Social en jóvenes universitarios argentinos. REVISTA DE PSICOLOGÍA, 7(14), 726.

Zubieta, E., Muratori , M., \& Fernández , O. (2012). Bienestar subjetivo y psicosocial: explorando diferencias de género. Salud \& Sociedad, 3(1), 66-76. doi:10.22199/s07187475.2012.0001.000 05 . 